\title{
Current issues in spinal anesthesia
}

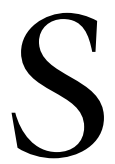

PINAL anesthesia is a venerable and simple technique. Technical competence is achieved early during training (>90\% technical success rate) after only 40-70 supervised attempts. ${ }^{1}$ Although the ease and long history of spinal anesthesia may give the impression that it is an unsophisticated technique, much has been recently learned regarding the anatomy, physiology, pharmacology, and applications of spinal anesthesia.

\section{Anatomy}

The arachnoid membrane is an important structure, as spinal agents must be delivered within its confines. The arachnoid membrane is composed of overlapping layers of epithelial cells connected by tight junctions. This anatomic arrangement allows the arachnoid membrane, not the dura, to function as the principal meningeal barrier $(90 \%$ of resistance) to materials crossing in and out of the cerebrospinal fluid (CSF). The arachnoid membrane serves not only as a passive container of CSF but also actively processes and transports agents attempting to cross the meninges. Recent studies demonstrate that metabolic enzymes are expressed in the arachnoid membrane that can affect agents (e.g., epinephrine) and neurotransmitters important for spinal anesthesia (e.g., acetylcholine). ${ }^{2}$ Active transport of compounds across the arachnoid membrane occurs in the area of the neural root cuffs where unidirectional transport of materials from the CSF into the epidural space occurs and may contribute to clearance of spinal anesthesia agents. After injection of spinal anesthetics, dilution with the CSF occurs prior to arrival at effector sites in the CNS. Thus, individual variation in lumbosacral volumes of CSF and distribution within this volume will affect spinal anesthesia. Recent imaging with magnetic resonance demonstrates great variability between individuals in volume of lumbosacral CSF with a range of $28-81 \mathrm{~mL}$. Interestingly, obese individuals have substantially less CSF ( $10 \mathrm{~mL}$ less) that is partly due to compression of the neural foramina. Clinical correlation between volume of lumbosacral CSF and spinal anesthesia with hyperbaric lidocaine and isobaric bupivacaine is excellent with CSF accounting for $80 \%$ of the variability for peak block height and regression of sensory and motor block. $^{3}$ Unfortunately, volume of lumbosacral CSF does not correlate with external physical measurements other than weight. Thus, CSF volume cannot be easily estimated from physical examination and is not easily applied to the clinical setting. ${ }^{3}$

\section{Physiology} Cardiovascular

The most common serious side effects from spinal anesthesia are hypotension and bradycardia, and closed claims surveys of 40,000-550,000 spinal anesthetics indicate an incidence of cardiac arrest from $0.04-10 / 10,000 .^{4,5}$ Large surveillance studies typically observed incidences of hypotension around 33\% and bradycardia around $13 \%$ in non-obstetrical populations. ${ }^{4}$ Risk factors for hypotension in non-obstetrical populations include block height $\geq \mathrm{T} 5$, age $\geq 40$ yr, baseline systolic blood pressure $(\mathrm{SBP})<120$ $\mathrm{mmHg}$, and spinal puncture above $\mathrm{L} 3 \geq 4$. Risk factors for development of bradycardia in non-obstetrical populations include baseline heart rate $(\mathrm{HR})<60$ beats. $\mathrm{min}^{-1}$, ASA I, use of beta blockers, prolonged PR interval on electrocardiogram, and block height $\geq$ T5. Analysis of closed claims for cardiac arrest during spinal anesthesia indicated that administration of sedation to produce a sleep-like state without spontaneous verbalization and lack of early administration of epinephrine were common patterns of management in cases of cardiac arrest. ${ }^{5}$

Cardiovascular effects of spinal anesthesia typically include a decrease in arterial blood pressure and central venous pressure (CVP) with only minor decreases in $\mathrm{HR}$, stroke volume, or cardiac output even in patients with poor left ventricular function (ejection fraction < $50 \%) .{ }^{6}$ Hypotension occurs from decreases in systemic vascular resistance (SVR) and CVP from sympathetic block with vasodilation and redistribution of central blood volume to lower extremities and splanchnic beds. ${ }^{6}$ This sympathetic block is rarely complete and

From the Departments of Anesthesiology, Virginia Mason Medical Center and the University of Washington, Seattle, USA.

Address correspondence to: Dr. Liu, Department of Anesthesiology, Virginia Mason Medical Center, 1100 Ninth Avenue, Mail Stop B2AN, Seattle, Washington, USA 98101. Phone: 206- 223-6980; Fax: 206-223-6982; E-mail: aness1@vmmc.org Financial support: Funded by the Department of Anesthesiology, Virginia Mason Medical Center. 
some preservation of sympathetic reflexes to stressful challenge typically occurs. Sudden bradycardia can occur from shift in cardiac autonomic balance towards the parasympathetic system as evidenced in spectral analysis of heart rate variability, from activation of left ventricular mechanorecptors from a sudden decrease in left ventricular volume (Bezold Jarisch reflex), or from increases in baroreflex activity. ${ }^{5}$

Prophylactic measures to prevent hypotension include prehydration with crystalloids or colloids or administration of vasoactive agents. On the whole, prehydration with crystalloids $(250-2000 \mathrm{~mL}$ ) appears to temporarily increase preload and cardiac output without consistently increasing arterial blood pressure or preventing hypotension. ${ }^{4}$ Pharmacokinetics of crystalloid explain its poor efficacy, as crystalloids are quickly re- distributed from the intravascular to the extravascular space. Administration of large volumes (> l L) of a crystalloid does not appear to confer additional benefit over small volumes $(250 \mathrm{~mL})$, and may be detrimental to patients with limited cardiopulmonary reserve. Prehydration with a colloid $(\geq 500 \mathrm{~mL})$ appears to be more effective than with a crystalloid at maintaining arterial blood pressure and perhaps decreasing the incidence of hypotension depending on definition and population. ${ }^{7}$ In contrast to prophylaxis, treatment of hypotension during spinal anesthesia will be effective with crystalloid or colloid due to changes in kinetics induced by spinal anesthesia and intravascular hypovolemia. ${ }^{8}$ Both clinical scenarios alter kinetics of cystalloid and colloid to allow retention within the intravascular space. Prophylactic administration of pharmacologic agents may be more effective than prehydration for prevention of hypotension. ${ }^{9}$ Alphaadrenergic agonists (e.g., phenylephrine) reliably increase arterial blood pressure by increasing SVR, however heart rate and cardiac output may decrease due to increased afterload. Mixed alpha and betaadrenergic agents (e.g., ephedrine) are also effective for increasing arterial blood pressure and preventing hypotension but act by primarily increasing heart rate and cardiac output with a smaller increase in SVR. ${ }^{6}$ These different physiologic mechanisms for alpha vs mixed alpha and beta-adrenergic agents also occur during treatment of hypotension during spinal anesthesia. ${ }^{6}$

\section{Thermoregulation}

Mild perioperative hypothermia is associated with an increased incidence of myocardial ischemia, cardiac morbidity, wound infection, blood loss and transfusion requirements. Spinal anesthesia will predictably cause core hypothermia within 30-60 min, and patients should be monitored and actively warmed if needed.
There are three main mechanisms for development of core hypothermia after spinal anesthesia. ${ }^{10}$ The first is redistribution of central heat to the periphery due to vasodilation from sympathetic block. This effect is maximal during the first $30-60 \mathrm{~min}$, causes a decrease in core temperature of about $1-2^{\circ} \mathrm{C}$, and depends on extent of sensory block and patient age. The second mechanism is loss of thermoregulation characterized by reduced shivering and vasoconstriction thresholds during spinal anesthesia. Finally, with loss of thermoregulatory vasoconstriction below the level of the sympathetic block, there is increased heat loss from vasodilation. If hypothermia develops, patients should be rewarmed, and spinal anesthesia accelerates rewarming compared to general anesthesia due to the residual sympathetic block and vasodilation. ${ }^{10}$

\section{Hypnotic effects}

There has been a recent convergence in mechanisms of general and spinal anesthesia. MAC, a traditional measure of inhalation agent potency for depth of anesthesia appears to have a primary mechanism in the spinal cord. ${ }^{11}$ In contrast, central neuraxial anesthesia may have direct effects on suppression of consciousness, and multiple studies have observed that patients appear drowsy after spinal anesthesia despite the lack of sedative medications. ${ }^{12}$ Correspondingly, both spinal and epidural anesthesia reduce the hypnotic requirements of midazolam, isoflurane, sevoflurane, and thiopental in surgical patients and laboratory studies. ${ }^{11}$ Possible mechanisms for hypnosis during spinal anesthesia include rostral spread of local anesthetics or decrease in reticular activating system activity due to interruption of afferent input. Animal models support the latter, as spinal anesthesia in rats decreases hypnotic requirements of thiopental without detection of local anesthetic in the brain or cervical spinal cord. In humans, the degree of sedation due to spinal anesthesia is related to peak block height with greater sedation observed with greater block heights. ${ }^{12}$ Clinical relevance for these observations is the decreased need for pharmacologic sedation with the use of spinal anesthesia.

\section{Clinical applications \\ Ambulatory anesthesia}

Spinal lidocaine has been a popular choice for ambulatory spinal anesthesia, and recent studies have examined dose response effects of lidocaine on anesthesia and recovery (Table). ${ }^{4}$ Transient neurologic symptoms (TNS) are clearly associated with use of spinal lidocaine with an approximate incidence of $20 \%$ in the ambulatory setting. ${ }^{13}$ Concern over the potential for 
TABLE Typical dose response effects of spinal local anesthetics for ambulatory anesthesia

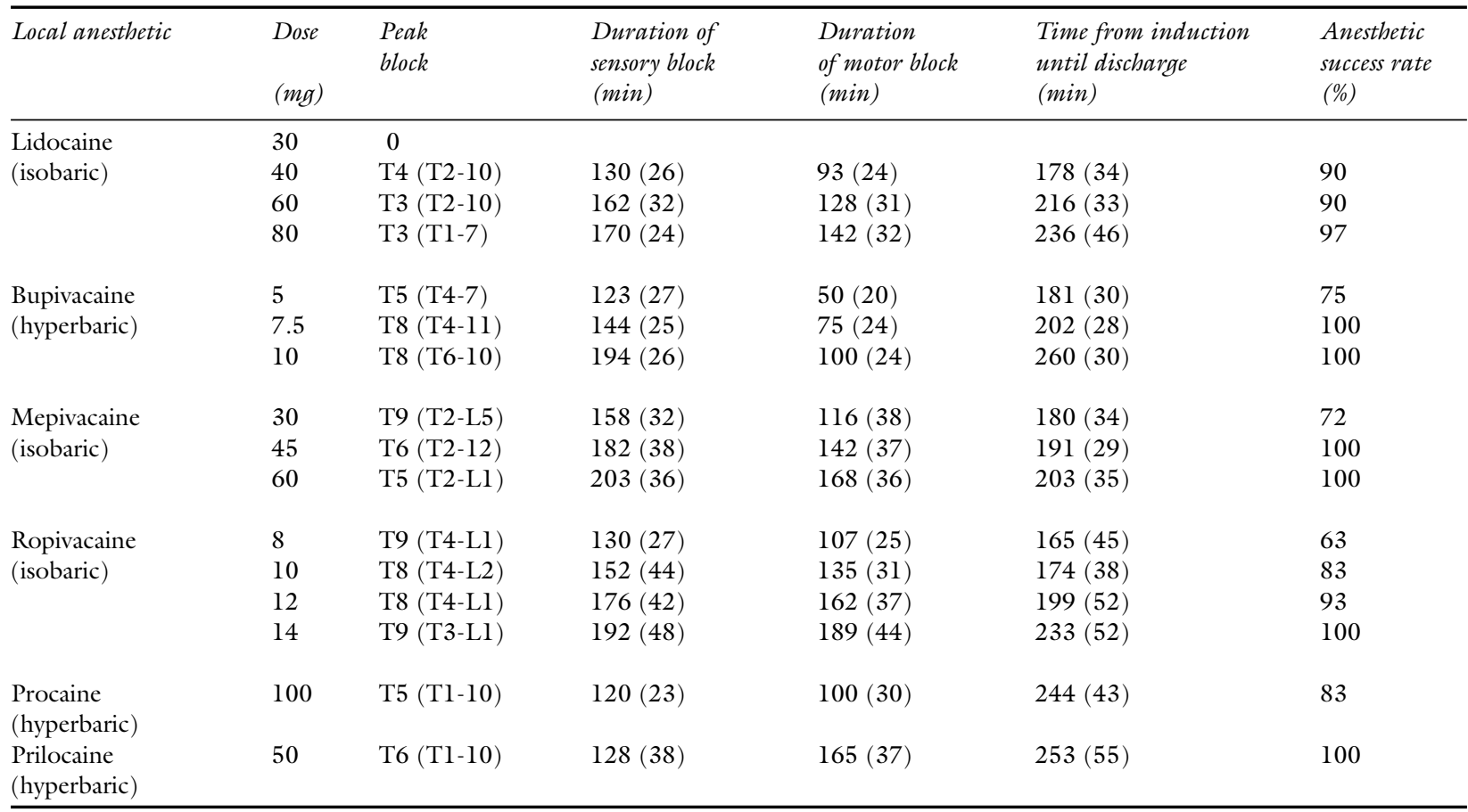

neurologic injury and for patient comfort has led to interest in alternative spinal local anesthetics. Bupivacaine has been the most studied alternative to lidocaine. TNS is virtually absent in all clinical studies with spinal bupivacaine $(0-1 \%) .{ }^{14}$ Recent doseresponse data on clinical anesthetic characteristics for spinal bupivacaine (Table) indicate that small doses can be used for ambulatory anesthesia. ${ }^{4}$ It is particularly important to select small doses of bupivacaine $(\leq$ $10 \mathrm{mg}$ ) to avoid prolonged detrusor block, inability to void, and excessively prolonged time until discharge as compared to equipotent doses of lidocaine. Mepivacaine has anesthetic characteristics similar to lidocaine with an approximate potency of 1.3:1 (Table). ${ }^{15}$ Reported risk of TNS with mepivacaine is highly variable. Small-scale studies (60-75 patients) report a low incidence of TNS (0-8 \%), whereas larger studies $(200+$ patients $)$ report incidences of $\sim 30 \%{ }^{4}$ It seems mepivacaine has similar clinical characteristics as lidocaine for spinal anesthesia but likely shares the same risk of TNS. Ropivacaine is approximately 50 to $60 \%$ as potent as spinal bupivacaine. Like bupivacaine, there is little risk of TNS with the use of spinal ropivacaine $(0-1 \%$ incidence) and, in equipotent doses (2:1), it will be virtually indistinguishable from bupivacaine for clinical anesthesia and risk of TNS without any obvious advantages. ${ }^{16}$ Spinal procaine appears to be less reliable for surgical anesthesia than lidocaine while having a slower recovery (Table). Risk of TNS with procaine $(\sim 6 \%)$ is less than with lidocaine but probably greater than with bupivacaine. ${ }^{17}$ Prilocaine is approximately equipotent to lidocaine within a dose range of 40-70 $\mathrm{mg}^{4}$ and thus may have suitable clinical characteristics for ambulatory spinal anesthesia (Table). Risk of TNS appears to be minimal with spinal prilocaine $(0-1 \%)$.

Both anesthetic success and especially time until ready for discharge are dependent on dose of local anesthetic. There has been recent interest in using analgesic additives to spinal local anesthetics to decrease the dose of local anesthetic for faster recovery while maintaining or improving anesthetic success. Addition of vasoconstrictors (epinephrine and phenylephrine) are effective for prolonging and intensifying spinal anesthesia but are ill advised for ambulatory surgery due to delay in patient recovery and potential increased risk of TNS. ${ }^{4}$ Numerous clinical studies have demonstrated that addition of 10-25 $\mathrm{\mu g}$ of fentanyl improves success of spinal anesthesia, allows use of less local anesthetic, and does not prolong duration until discharge. For example, $10 \mu \mathrm{g}$ of fentanyl added to $5 \mathrm{mg}$ of hyperbaric bupivacaine for outpatient knee arthroscopy improved anesthetic success from $75 \%$ with plain bupivacaine to $100 \%{ }^{4}$ 
A dose of $7.5 \mathrm{mg}$ plain bupivacaine is needed to achieve similar success with resultant prolongation of time until discharge of 187 to 202 min when compared to $5 \mathrm{mg}+$ fentanyl. Similar findings have been observed with addition of 10-25 $\mu \mathrm{g}$ of fentanyl to spinal lidocaine in patients undergoing ambulatory laparoscopy, in vitro fertilization, and knee arthroscopy. Addition of $25 \mu \mathrm{g}$ fentanyl to $20 \mathrm{mg}$ spinal lidocaine in patients having knee arthroscopy provides comparable anesthesia to $50 \mathrm{mg}$ lidocaine while providing shorter discharge times (145 vs 180 min) and reduced incidence of TNS (4\% vs 33\%). ${ }^{18}$ Indeed, discharge times after such small doses of local anesthetic are comparable to local anesthesia/propofol infusion. ${ }^{19}$ Dose response data for spinal clonidine suggests that a dose of $15-45 \mu \mathrm{g}$ is an optimal dose for low dose outpatient spinal anesthesia. This dose improved anesthetic success of $8 \mathrm{mg}$ ropivacaine from $60 \%$ to $100 \%$ for ambulatory knee arthroscopy without prolonging recovery. ${ }^{20}$

\section{TNS}

Prospective randomized studies reveal an incidence of TNS after lidocaine spinal anesthesia between $4-33 \%{ }^{13}$ Risk of TNS is increased with use of lidocaine, ambulatory anesthesia, lithotomy and knee arthroscopy positions and is unaffected by baricity or dilution of lidocaine to $0.5 \%{ }^{13}$ TNS typically occur 12-36 hr after resolution of spinal anesthesia, last for two to three days, and is typically rated as a $3-4 / 10$ for pain intensity $(0=$ no pain, $10=$ worst pain $)$. Discomfort from TNS is self-limited and can be treated with potent non-steroidal anti-inflammatory drugs (NSAIDs) and trigger point injections. ${ }^{21}$

Neurotoxic etiology for TNS remains speculative. Patients reporting TNS do not develop sensory or motor deficits in contrast to cauda equina syndrome. Imaging of the CNS does not show evidence of injury to spinal cord or nerve roots in patients with TNS. Sensitive measures of neural electrophysiology (SSEP, EMG, nerve conduction velocity, $\mathrm{H}$ reflex, $\mathrm{F}$ waves) do not change during TNS as compared to before spinal anesthesia. ${ }^{22}$ Laboratory work in both intrathecal and desheathed peripheral nerve models indicate that concentration of lidocaine is a critical factor in the neurotoxicity ${ }^{21}$ of desheathed peripheral nerves. Yet, clinical trials report high incidences of TNS (17\%) with spinal injection of very dilute lidocaine concentrations $(0.5 \%, 1 \%)^{13}$ that touch upon the minimal effective concentration for spinal lidocaine $(0.0-07 \%)$. Indeed, further dilution of lidocaine should occur due to active mixing in spinal CSF after non-preferential distribution of hyperbaric solution with typical, clini- cal use of small gauge pencil point needles. These clinical observations lessen the plausibility of a concentration dependent neurotoxic etiology. Finally, successful treatment of TNS with trigger point injections and NSAIDs also fail to substantiate neurologic injury as an etiology. All together, these data may indicate a myofascial etiology for the radiating backpain, and some experts have called for a change in nomenclature from TNS to postspinal musculoskeletal symptoms. ${ }^{23}$

\section{Low molecular weight heparin (LMWH)}

Anticoagulants are frequently used in the surgical population as prophylaxis and treatment for thrombotic conditions. Analysis of closed claims for neurologic injury indicates that anti-coagulation is a major risk factor for spinal cord injury with spinal anesthesia. ${ }^{24}$ LMWH is a fractionated component of standard heparin and has become a popular agent. LMWH has much greater bioavailability than unfractionated heparin, primarily affects coagulation factor $\mathrm{X}$, and cannot be monitored with partial thromboplastin time. ${ }^{25}$ Over a decade of European experience suggested that perioperative use of LMWH did not add substantial risk to spinal anesthesia. However, the US experience has been different with $>40$ cases of spinal hematomas since its introduction in 1993. In contrast to European experience of relative safety, estimated risk of spinal hematoma with $\mathrm{LMWH}$ and spinal anesthesia in the US is $1: 41,000$ vs the $1: 225,000$ in the non-anticoagulated patient. ${ }^{25}$ Larger daily doses and more frequent administration with US practice may account for this apparent increase in risk. Guidelines to safe use of LMWH and spinal anesthesia include delay of administration of LMWH for 12-24 hr after spinal puncture. If the patient is already using LMWH, then it should be stopped for at least 12-24 hr prior to spinal puncture. ${ }^{25}$

\section{Conclusion}

Spinal anesthesia is an old, simple, and popular anesthetic technique, yet much remains unknown regarding pertinent anatomy, physiology, and pharmacology. Investigations into physiologic effects of spinal anesthesia reveal complex actions on multiple organ systems. New local anesthetics, analgesic additives, and techniques are being investigated for different applications as the practice of medicine focuses on outpatient care. Safety of spinal agents and complications from spinal anesthesia continue to be examined and re-examined to improve safety. Further study will be needed to fully resolve these issues and to further understand and improve the clinical use of spinal anesthesia. 


\section{References}

1 Konrad C, Schupfer G, Wietlisbach M, Gerber $H$. Learning manual skills in anesthesiology: is there a recommended number of cases for anesthetic procedures? Anesth Analg 1998; 86: 635-9.

2 Ummenhofer WC, Brown SM, Bernards CM. Acetylcholinesterase and butyrylcholinesterase are expressed in the spinal meninges of monkeys and pigs. Anesthesiology 1998; 88: 1259-65.

3 Carpenter RL, Hogan QH, Liu SS, Crane B, Moore J. Lumbosacral cerebrospinal fluid volume is the primary determinant of sensory block extent and duration during spinal anesthesia. Anesthesiology 1998; 89: 24-9.

4 Liu SS, McDonald SB. Current issues in spinal anesthesia. Anesthesiology 2001; 94: 888-906.

5 Pollard JB. Cardiac arrest during spinal anesthesia: common mechanisms and strategies for prevention. Anesth Analg 2001; 92: 252-6.

6 Butterworth J. Physiology of spinal anesthesia: what are the implications for management? Reg Anesth Pain Med 1998; 23: 370-3; Discussion 384-7.

7 Ueyama H, Yan-Ling H, Tanigami H, Mashimo T, Yoshiya I. Effects of crystalloid and colloid preload on blood volume in the parturient undergoing spinal anesthesia for elective cesarean section. Anesthesiology 1999; 91: 1571-6.

8 Hahn RG, Resby M. Volume kinetics of Ringer's solution and dextran 3\% during induction of spinal anaesthesia for caesarean section [published erratum appears in Can J Anaesth 1998; 45: 1223-4]. Can J Anaesth 1998; 45: 443-51.

9 Chan WS, Irwin MG, Tong WN, Lam $\Upsilon$ H. Prevention of hypotension during spinal anaesthesia for caesarean section: ephedrine infusion versus fluid preload. Anaesthesia 1997; 52: 908-13.

10 Sessler DI. Perioperative heat balance. Anesthesiology 2000; 92: 578-96.

11 Hodgson PS, Liu SS, Gras TW. Does epidural anesthesia have general anesthetic effects? Anesthesiology 1999; 91: 1687-92.

12 Pollock JE, Neal JM, Liu SS, Burkhead D, Polissar N. Sedation during spinal anesthesia. Anesthesiology 2000; 93: 728-34.

13 Pollock JE, Liu SS, Neal JM, Stephenson CA. Dilution of spinal lidocaine does not alter the incidence of transient neurologic symptoms. Anesthesiology 1999; 90: 445-50.

14 Freedman JM, Li DK, Drasner K, Jaskela MC, Larsen $B$, Wi S. Transient neurologic symptoms after spinal anesthesia: an epidemiologic study of 1,863 patients. Anesthesiology 1998; 89: 633-41.

15 Zayas VM, Liguori GA, Chisolm MF. Dose response relationships for isobaric spinal mepivacaine using the combined spinal epidural technique. Anesth Analg 1999; 89: 167-71.

16 Gautier PE, DeKock MF, Van Steenberge A. Intrathecal ropivacaine for ambulatory surgery: a comparison between intrathecal bupivacaine and intrathecal ropivacaine for knee arthroscopy. Anesthesiology 1999; 91: 1239-45.

17 Hodgson PS, Liu SS, Batra MS, Gras TW, Pollock JE, Neal JM. Procaine compared to lidocaine for incidence of transient neurologic symptoms. Reg Anesth Pain Med 2000; 25: 218-22.

18 Ben-David B, Maryanovsky $M$, Gurevitch A, et al. A comparison of minidose lidocaine-fentanyl and conventional-dose lidocaine spinal anesthesia. Anesth Analg 2000; 91: 865-70.

19 Ben-David B, DeMeo PJ, Lucyk C, Solosko D. A comparison of minidose lidocaine-fentanyl spinal anesthesia and local anesthesia/propofol infusion for outpatient knee arthroscopy. Anesth Analg 2001; 93: 319-25.

20 De Kock M, Gautier P, Fanard L, Hody JL, Lavand'homme $P$. Intrathecal ropivacaine and clonidine for ambulatory knee arthroscopy: a dose-response study. Anesthesiology 2001; 94: 574-8.

21 Hodgson PS, Neal JM, Pollock JE, Lim SS. The neurotoxicity of drugs given intrathecally (spinal). Anesth Analg 1999; 88: 797-809.

22 Pollock JE, Burkhead D, Neal JM, et al. Spinal nerve function in five volunteers experiencing neurologic symptoms after lidocaine subarachnoid anesthesia. Anesth Analg 2000; 90: 658-65.

23 Rowlingson JC. To avoid "transient neurologic symptoms" - The search continues. RAPM 2000; 25 : 215-7.

24 Cheney FW, Domino KB, Caplan RA, Posener K. Nerve injury associated with anesthesia. A closed claims analysis. Anesthesiology 1999; 90: 1062-9.

25 Horlocker TT, Wedel DJ. Neurologic complications of spinal and epidural anesthesia. Reg Anesth Pain Med 2000; 25: 83-98. 